\title{
Propriedades mecânicas de aloenxertos meniscais de coelhos após preservação em glicerina e reidratação em $\mathrm{NaCl} 0,9 \%$
}

\author{
Mechanical properties of rabbit meniscal allografts after glycerin preservation and rehydration \\ in $\mathrm{NaCl} 0.9 \%$
}

\author{
Liana Mesquita Vilela ${ }^{\mathrm{I}}$ Ricardo Junqueira Del Carlo ${ }^{\mathrm{I}^{*}}$ Rubens Chaves de Oliveira ${ }^{\mathrm{II}}$ \\ Amanda Maria Sena Reis ${ }^{I}$ Ana Flora Sousa de Brito ${ }^{I}$ Edson Vilela de Melo Filho ${ }^{\text {III }}$ \\ Gustavo de Freitas Ferrari ${ }^{I}$ Simone Rezende Galvão ${ }^{I}$
}

\section{RESUMO}

Foram avaliadas as propriedades mecânicas, força, deformação e tensão ao limite elástico, tensão ao ponto de ruptura $e$ índice de rigidez, de meniscos frescos e meniscos transplantados por 70 dias em joelhos de coelhos, após preservação em glicerina. O primeiro grupo (tratado) foi formado por seis meniscos mediais do joelho esquerdo, coletados de animais oriundos de criação comercial. Esses meniscos foram armazenados em glicerina $98 \%$ por um período de 45-60 dias; em seguida, foram reidratados em solução salina a 0,5\% de enrofloxacina, por 12 horas, $e$ implantados em joelhos de outros seis coelhos, submetidos à meniscectomia. Após 70 dias da cirurgia, foi realizada a eutanásia dos animais e retirada dos aloenxertos para avaliação mecânica. $O$ segundo grupo (controle) foi composto por seis meniscos mediais, retirados dos joelhos contralaterais (direito) dos mesmos animais. Para a amostra estudada, não houve diferença estatisticamente significante entre os grupos controle e tratado para nenhuma das variáveis analisadas, demonstrando que a reidratação de aloenxertos meniscais, após preservação em glicerina, mantém as características mecânicas desses, após 70 dias de implantação, semelhantes às de meniscos frescos.

Palavras-chave: meniscos, enxertos homólogos, glicerina, joelho.

\section{ABSTRACT}

We evaluated the mechanical properties, strength, strain and stress to the elastic limit, the rupture stress point and stiffness index of fresh menisci and menisci transplanted for 70 days in the knees of rabbits after preservation in glycerin. The first group (treated) was formed by six medial menisci of the left knee, collected from these animals for commercial breeding. These menisci were stored in 98\% glycerin for a period of $30-60$ days, and then rehydrated in a solution in $0.5 \%$ saline enrofloxacin for 12 hours, and implanted in the knees of another six rabbits underwent meniscectomy. After 70 days of surgery was performed the euthanasia of animals and removal of allografts for mechanical evaluation. The second group (control) consisted of six medial menisci, removed from the contralateral knees (right) of the same animals. For the sample studied, had no statistically significant difference between control and treated groups for any of the variables analyzed, showing that the rehydration of meniscal allografts after preservation in glycerin keeps the mechanical characteristics of these, after 70 days of implantation, similar to the meniscus fresh.

Key words: menisci, allografts, glycerin, knee.

\section{INTRODUÇÃO}

Os meniscos são estruturas fibrocartilaginosas, semicirculares, moldáveis, localizadas entre os côndilos femorais e o platô tibial. Suas funções incluem a distribuição de forças, absorção de choques, estabilidade articular, proteção e lubrificação das superfícies articulares e nutrição da cartilagem (HULSE \& JOHNSON, 2002; RIJK, 2004). A borda periférica do menisco medial encontrase aderida à cápsula articular e ao ligamento colateral medial, reduzindo sua mobilidade em relação ao menisco lateral. A menor mobilidade meniscal e as cargas que a região medial do joelho recebe, tornam o menisco medial mais propenso às lesões durante movimentos rotacionais (McCARTY et al., 2002).

Segundo SOLOMON et al. (2002), na rotação interna da tíbia, o menisco medial vai para

\footnotetext{
IDepartamento de Veterinária (DVT), Universidade Federal de Viçosa (UFV), 36570-000, Viçosa, MG, Brasil. E-mail: ricarlo@ufv.br. *Autor para correspondência.

IIDepartamento de Engenharia Florestal, Laboratório de Celulose e Papel, UFV, Viçosa, MG, Brasil.

IIIDepartamento de Veterinária, Universidade Federal Rural de Pernambuco (UFRPE), Recife, PE, Brasil.
} 
frente e o lateral vai para trás, enquanto, na rotação externa, acontece o contrário, ou seja, acompanham o movimento do fêmur. Isso ocorre devido à ação de tracionamento dos ligamentos alares e à pressão que o fêmur exerce sobre os meniscos. Durante a flexão, os meniscos são tracionados para trás e, durante a extensão, para frente, conforme suas inserções. Na flexão extrema, a porção caudal do menisco é firmemente comprimida entre a porção posterior do platô tibial e o côndilo femoral. A maioria das lacerações meniscais que provocam claudicação em cães ocorre em conjunto com rupturas do ligamento cruzado cranial (HULSE \& JOHNSON, 2002).

A cicatrização do menisco ocorre apenas quando a lesão é na região periférica, local em que existe suprimento sanguíneo (MCCARTY et al., 2002). Os métodos de tratamento das lesões meniscais incluem o reparo primário, a meniscectomia parcial e a total. O reparo ou sutura é indicado quando as lesões são agudas, longitudinais e ocorrem em áreas vascularizadas (CANNON, 1996). Apenas 30 a $50 \%$ das lesões podem ser tratadas dessa forma (FRIEDMAN, 2003; RECKERS et al., 2005).

Quando a lesão meniscal não é indicativa de reparo nem de meniscectomia parcial, optase pela meniscectomia total (VILELA et al., 2010a). FUKUBAYASHI \& KUROSAWA (1980) observaram diminuição de $50 \%$ da área de contato em joelhos submetidos à meniscectomia total e aumento significativo na pressão por unidade de área, resultando em lesão da cartilagem articular (RECKERS et al., 2005).

Nesses casos, deve-se considerar a substituição dos meniscos por enxertos homólogos (CURY et al., 2002; WYLAND et al., 2002; RECKERS et al., 2005; VILELA et al., 2010a), enxertos autólogos e até por próteses (RECKERS et al., 2005), com o intuito de prevenir o comprometimento da cartilagem articular (WIRTH et al., 2002; RIJK, 2004). GAO et al. (1998), em estudos experimentais com coelhos, demonstraram que a fixação segura do aloenxerto é crítica para a cicatrização inicial, remodelamento do enxerto e, a longo prazo, para suas funções.

$\mathrm{Na}$ literatura, são escassos os trabalhos que avaliam as propriedades mecânicas de meniscos. Sendo assim, o objetivo deste estudo foi avaliar a resistência à compressão de aloenxertos meniscais mediais de coelhos, após preservação em glicerina e reidratação em $\mathrm{NaCl}$ 0,9\%.

\section{MATERIAL E MÉTODOS}

Foram utilizados 12 coelhos albinos, machos, adultos, da raça Nova Zelândia, com peso médio de $4 \mathrm{~kg}$. Durante o período do experimento, os animais foram alojados em gaiolas individuais, em temperatura ambiente e alimentados com ração própria para a espécie e água à vontade. O experimento foi dividido em três etapas. Na primeira etapa, foi realizada a coleta de meniscos mediais do joelho esquerdo de seis coelhos provenientes de criação comercial. Após a coleta, os meniscos foram armazenados, submersos, em frascos individuais contendo glicerina $98 \%$ por um período de 45 a 60 dias.

Na segunda etapa, que ocorreu de 3060 dias após a coleta, os meniscos conservados em glicerina 98\% foram reidratados, por 12 horas, em solução salina a $0,5 \%$ de enrofloxacina, e implantados em joelhos de outros seis coelhos, após meniscectomia medial total no joelho esquerdo. Na terceira etapa, 70 dias após a implantação, os coelhos foram submetidos à eutanásia. O menisco implantado e o medial contralateral foram removidos e submetidos ao teste mecânico de compressão. O menisco medial do joelho esquerdo foi considerado grupo tratado (GT) e o do joelho direito grupo controle (GC).

No pré-operatório, os animais foram sedados com acepromazina $0,2 \%(0,5 \mathrm{mg} / \mathrm{kg}, \mathrm{IM})$ e receberam anti-inflamatório, meloxicam $0,2 \%\left(0,2 \mathrm{mg} \mathrm{kg}^{-1}, \mathrm{SC}\right)$ e antibioticoterapia com enrofloxacina 2,5\% (10 $\mathrm{mg} \mathrm{kg}^{-1}$, SC). A anestesia foi obtida com a associação midazolam (3mg kg-1) e cetamina (25mg kg-1), por via intramuscular, e mantida com isoflurano em máscara de oxigênio em fluxo constante de 2,5L min ${ }^{-1}$.

Foi realizada incisão longitudinal de pele e tecido subcutâneo na superfície crânio-medial do joelho, com cerca de seis centímetros de extensão. Em seguida, o tecido subcutâneo foi dissecado até a exposição medial da cápsula articular, que foi incisada juntamente com a membrana sinovial. A patela foi luxada lateralmente, o joelho flexionado e a articulação exposta. Um discreto esforço em valgo com o joelho flexionado foi suficiente para permitir a abordagem ao menisco medial. A meniscectomia total medial foi realizada após secção dos ligamentos meniscocapsulares e meniscotibiais, que foi obtida com auxílio de bisturi com lâmina no 15 e agulha hipodérmica 40x12.

Previamente à implantação, cada menisco recebeu dois pontos simples separados com auxílio de uma agulha de insulina, utilizando o fio poliglactina 910, $n^{\circ}$ 6.0. O primeiro na região entre o corno cranial e o terço médio do menisco e o segundo entre o terço médio e o corno caudal (Figura $1 \mathrm{~A}$ ). O menisco foi então posicionado sobre o platô tibial e os fios passados no sentido do interior para o exterior da 
cápsula articular. Em seguida, a pele foi afastada, possibilitando a visualização dos fios (Figura 1 B), aplicação de nós sobre o tecido subcutâneo e a fixação do menisco.

Posteriormente, a patela foi reposicionada, a cápsula articular e tecido subcutâneo suturados com pontos isolados simples e fio poliglactina 910, $\mathrm{n}^{\mathrm{o}} 4.0$, e a pele com pontos isolados simples e fio mononáilon $n^{-}$4.0. No pós-operatório, os animais receberam enrofloxacina $(10 \mathrm{mg} \mathrm{kg}$, IM, a cada 24 horas) durante oito dias, meloxicam $\left(0,2 \mathrm{mg} \mathrm{kg}^{-1}\right.$, SC, a cada 24 horas), durante quatro dias e sulfato de morfina (1,0mg kg-1, SC, a cada 6 horas) durante dois dias. A eutanásia dos coelhos foi realizada 70 dias após a implantação, onde os aloenxertos e os meniscos mediais contralaterais foram retirados, e submetidos à avaliação mecânica.

Os testes foram realizados em sala climatizada, com umidade relativa do ar de $50 \pm 2 \%$ e temperatura de $23 \pm 1^{\circ} \mathrm{C}$, em equipamento Instron modelo 4204, com capacidade da célula de carga de $1000 \mathrm{~N}$, precisão de $\pm 0,2 \mathrm{~N}$ e aquisição de 20 pontos de força e deformação por segundo. A velocidade de aplicação de força ocorreu numa taxa de $25 \mathrm{~mm}$ minuto $^{-1}$ até a ruptura total de cada amostra.

O menisco foi colocado no equipamento, na superfície da barra de compressão inferior, com uma distância de 3mm entre as barras superior e inferior. A carga foi aplicada até as barras ficarem a uma distância de $1 \mathrm{~mm}$, o que acarretou no esmagamento total da amostra. O valor da força e deformação ao limite de elasticidade foi registrado em Newton e milímetros, respectivamente.

A leitura dos valores das forças aplicadas foi realizada com a utilização de célula de carga e a determinação das deformações foi realizada pelo deslocamento da barra de compressão do referido equipamento; ambos conectados a um sistema de aquisição de dados controlados por computador, que permitiu o controle automatizado do sistema responsável pela coleta de dados e derivação das propriedades analisadas.

As propriedades avaliadas foram: 1) força ao limite de elasticidade: força absorvida pelo corpo de prova no seu limite máximo de elasticidade, ou seja, até essa força, o corpo de prova ainda tem capacidade de retornar a sua estrutura original, sem sofrer deformação permanente (deformação inelástica), expressa em Newton; 2) deformação ao limite de elasticidade: deformação sofrida durante a aplicação da força, que desaparece retornando a sua dimensão original, após a retirada dessa força; 3) tensão ao limite de elasticidade: razão da força ao limite de elasticidade pela área do corpo de prova; 4) tensão ao ponto de ruptura: razão da força ao ponto de ruptura pela área do corpo de prova; 5) índice de rigidez (módulo de elasticidade ou módulo de Young): expressa a habilidade do corpo de prova em resistir às deformações em função das tensões aplicadas, expresso em $\left(\mathrm{N} \mathrm{m}^{-2}\right)$. É a medida de rigidez de um material sólido, ou seja, quanto maior, menor é a deformação sofrida pelo material e, portanto, mais o material resiste às forças aplicadas.

Para a comparação das variáveis, força ao limite de elasticidade, deformação ao limite de elasticidade, tensão ao limite de elasticidade e ao ponto de ruptura e índice de rigidez, entre os grupos controle e tratado, foi aplicado o teste não paramétrico de Mann-Whitney, devido ao número pequeno de observações. Valores de $\mathrm{P}<0,05$ indicam que existe diferença estatisticamente significante entre os grupos. O nível de significância assumido foi de $5 \%$. Os cálculos estatísticos foram realizados no

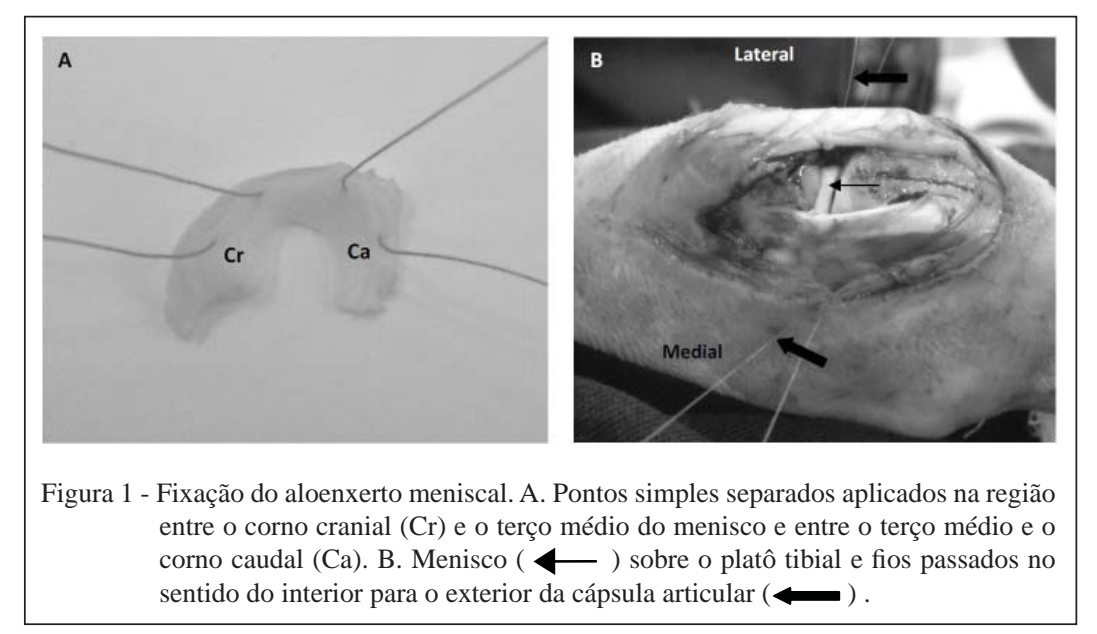

Ciência Rural, v.43, n.12, dez, 2013. 
software SPSS v18.0. Cada grupo foi composto por seis meniscos.

\section{RESULTADOS E DISCUSSÃO}

Na técnica cirúrgica utilizada neste estudo, o menisco transplantado foi fixado à cápsula articular por meio de duas suturas passadas no menisco e depois do interior para o exterior da articulação, com fio de poliglactina 910 nํㅜ 6.0. CURY et al. (2002) utilizaram técnica semelhante, mas com fio de náilon nº 6.0 e também obtiveram fixação estável, que é uma etapa importante para o sucesso do transplante. Em um estudo com coelhos, GAO et al. (1998) demonstraram que a fixação segura do aloenxerto é fundamental para a cicatrização inicial, remodelamento do enxerto e, a longo prazo, para suas funções.

A força absorvida pelo menisco no seu limite máximo de elasticidade, ou seja, quando o menisco mantém a capacidade de retornar a sua estrutura original após a retirada da força, foi estatisticamente semelhante entre o grupo controle e o grupo de meniscos transplantados, indicando que os dois grupos foram capazes de suportar forças compressivas de forma semelhante, antes de sofrerem ruptura e consequente deformação inelástica (permanente).

Dessa maneira, pode-se afirmar que a preservação dos meniscos em glicerina 98\% por um período de 30-60 dias e a reidratação em solução salina por 12 horas não alterou essa propriedade mecânica nos aloenxertos após 70 dias do transplante; fato também relatado por VILELA et al. (2010b), ao estudarem as propriedades mecânicas de meniscos preservados de coelhos, mas sem a implantação.
A força ao limite de elasticidade média no grupo controle foi de $161,49 \mathrm{~N}$, enquanto, no grupo tratado, foi de 137,03N (Tabela 1). Alterações da força ao limite elástico, dos aloenxertos meniscais, pode interferir negativamente em determinadas funções, como na absorção de choques, distribuição de forças e proteção das cartilagens articulares (RIJK, 2004).

O valor médio da deformação ao limite de elasticidade do grupo de meniscos controle e de meniscos transplantados foi de 1,61\% e 2,0\%, respectivamente, (Tabela 1) não caracterizando diferença estatística, ou seja, a deformação elástica (reversível) sofrida pelos meniscos após 70 dias de implantação foi semelhante à dos meniscos frescos. Esse resultado é semelhante ao encontrado por VILELA et al. (2010b), que não observaram diferenças entre o grupo de meniscos controles e meniscos preservados em glicerina e reidratados em solução $\mathrm{NaCl}$ 0,9\%.

De acordo com RECKERS et al. (2005) e VILELA et al. (2010b), a glicerina 98\% desidrata o tecido, tornando-o mais rígido e menos elástico, diminuindo sua capacidade de deformação reversível. Esses autores comprovaram a importância da reidratação de aloenxertos, após preservação em glicerina, quando demonstraram que meniscos de coelhos, preservados nesse meio e não reidratados, apresentaram menor capacidade de deformação elástica, quando comparados com meniscos frescos e meniscos preservados e reidratados em solução $\mathrm{NaCl}$ 0,9\%.

Dessa forma, a reidratação dos meniscos em solução de $\mathrm{NaCl}$ 0,9\% por 12 horas, após preservação em glicerina, realizada neste estudo, conforme recomendado por DEL CARLO et

Tabela 1 - Valores da média, desvio padrão, valor mínimo e máximo das propriedades mecânicas (força ao limite de elasticidade, deformação ao limite de elasticidade e tensão ao limite de elasticidade) analisadas no teste de compressão dos meniscos do grupo controle e do grupo tratado. Os dois grupos não diferem entre si em nível de 5\% de probabilidade.

\begin{tabular}{|c|c|c|c|c|c|c|}
\hline & Grupo & Média & DP & Mínimo & Máximo & $\mathrm{P}$ \\
\hline \multirow{2}{*}{ Força ao limite de elasticidade (N) } & Controle & 161,49 & 58,57 & 104,59 & 270,74 & \multirow{2}{*}{0,485} \\
\hline & Tratado & 137,03 & 53,57 & 83,17 & 214,49 & \\
\hline \multirow{2}{*}{ Deformação ao limite de elasticidade (\%) } & Controle & 1,61 & 0,34 & 1,31 & 2,21 & \multirow{2}{*}{0,126} \\
\hline & Tratado & 2,00 & 0,39 & 1,45 & 2,42 & \\
\hline \multirow{2}{*}{ Tensão ao limite de elasticidade (MPa) } & Controle & 1,83 & 0,55 & 1,25 & 2,79 & \multirow{2}{*}{0,818} \\
\hline & Tratado & 1,79 & 0,67 & 1,06 & 2,68 & \\
\hline
\end{tabular}

DP: Desvio Padrão. 
al. (1999), foi imprescindível, pois possibilitou aos aloenxertos meniscais, implantados por 70 dias, manter a capacidade de deformação elástica semelhante aos meniscos contralaterais, recuperando assim as características e a função fisiológica alteradas pela glicerina. Resultados semelhantes foram relatados por PIGOSSI (1967), após reidratar dura-máter canina e humana preservadas em glicerina $98 \%$ por sete meses.

A tensão ao limite de elasticidade, suportada pelo grupo de meniscos preservados, reidratados e transplantados e pelo grupo de meniscos frescos (grupo controle), foi semelhante, não havendo diferença significativa entre os dois grupos. Corroborando os achados de VILELA et al. (2010b), que observaram que os meniscos reidratados suportaram a tensão quando submetidos a uma força capaz de promover deformação elástica, da mesma forma que os meniscos frescos, demonstrando que a reidratação em $\mathrm{NaCl}$ 0,9\% é necessária para recuperar a elasticidade dos enxertos após o período de preservação em glicerina.

Pode-se afirmar que, após 70 dias de implantação, os aloenxertos meniscais mantiveram a tensão ao limite elástico, igual aos meniscos do grupo controle. Neste, a tensão média ao limite de elasticidade foi de 1,83MPa e, no grupo dos aloenxertos meniscais, a média foi de 1,79MPa (Tabela 1).

Quanto à tensão ao ponto de ruptura, não houve diferença estatisticamente significante entre o grupo de meniscos implantados e o grupo de meniscos controle. Dessa forma, pode-se dizer que a preservação dos meniscos em glicerina, a reidratação e sua implantação por 70 dias, não alterou essa propriedade mecânica, sendo seu valor médio 2,90MPa no grupo tratado e 3,47MPa no grupo controle (Tabela 2). Esses resultados são semelhantes aos encontrados por VILELA et al. (2010b), que observaram ainda que os meniscos não reidratados, após preservação em glicerina 98\%, suportaram maior tensão no momento da ruptura, quando comparados com os meniscos frescos e os meniscos reidratados, devido à menor elasticidade e a maior rigidez. Essas características são inapropriadas, funcionalmente, aos meniscos, que atuam como estruturas móveis e elásticas, promovendo efeito amortecedor e a congruência entre o fêmur e a tíbia (HULSE \& JOHNSON, 2002; TORRES et al., 2006).

O índice de rigidez dos meniscos do grupo controle e do grupo de aloenxertos foi estatisticamente igual. Isso significa que os meniscos dos dois grupos conseguiram resistir de forma semelhante às forças compressivas neles aplicadas. Neste estudo, após a preservação dos meniscos em glicerina, realizou-se a reidratação em $\mathrm{NaCl}$ 0,9\%, por doze horas, antes do transplante, conforme recomendado por VILELA et al. (2010b). Esses autores relataram que esse período de reidratação foi suficiente para diminuir a rigidez, aumentando assim a capacidade elástica dos meniscos. O índice de rigidez médio encontrado neste estudo foi de 113,95MPa e 88,80MPa, para os grupos de meniscos frescos e de meniscos transplantados, respectivamente (Tabela 2 ).

\section{CONCLUSÃO}

A análise de aloenxertos meniscais preservados em glicerina e reidratados em $\mathrm{NaCl}$ 0,9\%, realizada após 70 dias de implantação, demonstra que as variáveis mecânicas, força, deformação e tensão ao limite de elasticidade; tensão ao ponto de ruptura; e índice de rigidez comportam-se de forma semelhante às dos meniscos frescos, constituindo-se opção terapêutica após a meniscectomia medial em coelhos e para estudos clínicos em outras espécies animais.

Tabela 2 - Valores da média, desvio padrão, valor mínimo e máximo das propriedades mecânicas (tensão ao ponto de ruptura e índice de rigidez) analisadas no teste de compressão dos meniscos do grupo controle e do grupo tratado. Os dois grupos não diferem entre si em nível de $5 \%$ de probabilidade.

\begin{tabular}{|c|c|c|c|c|c|c|}
\hline & Grupo & Média & DP & Mínimo & Máximo & $\mathrm{P}$ \\
\hline \multirow{2}{*}{ Tensão ao ponto de ruptura (MPa) } & Controle & 3,47 & 0,60 & 2,31 & 3,85 & \multirow{2}{*}{0,173} \\
\hline & Tratado & 2,90 & 0,81 & 1,89 & 4,18 & \\
\hline \multirow{2}{*}{ Índice de rigidez - MOE (MPa) } & Controle & 113,95 & 24,19 & 85,46 & 145,97 & \multirow{2}{*}{0,179} \\
\hline & Tratado & 88,80 & 26,47 & 61,46 & 121,47 & \\
\hline
\end{tabular}

DP: Desvio Padrão. 


\section{COMITÊ DE ÉTICA E BIOSSEGURANÇA}

Parecer nº 89/2007.

\section{AGRADECIMENTOS}

O presente estudo foi financiado parcialmente pelo Conselho Nacional de Desenvolvimento Científico e Tecnológico (CNPq) e a Coordenação de Aperfeiçoamento de Pessoal de Nível Superior (CAPES).

\section{REFERÊNCIAS}

CANNON W.D. Arthroscopic meniscal repair. In: MCGINTY, J.B. et al. Operative arhroscopy. Philadelphia, PA: Lippincott Willinas \& Wilkins, 1996, p.299-315.

CURY, R.P.L. et al. Transplante homólogo de menisco: estudo experimental em coelhos. Revista Brasileira de Ortopedia e Traumatologia, v.37, n.8, p.341-350, 2002. Disponível em: $<$ http://www.rbo.org.br/materia.asp?idIdioma $=1 \& \mathrm{mt}=783>$. Acesso em: 20 mar. 2011.

DEL CARLO, R.J. et al. Aloenxertos ósseos caninos diferentemente preservados. Revista Brasileira de Ciência Veterinária, v.6 n.3, p.121-126, 1999

FRIEDMAN, M.D. Meniscal Allografts. Arthroscopy, v.18, p.3339, 2003.

FUKUBAYASHI, T.; KUROSAWA, $\mathrm{H}$. The contact area and pressure distribution pattern of the knee: a study of normal and osteoarthritic knee joints. Acta Orthopaedica Scandinavica, v.52, p.871-879, 1980.

GAO, J. et al. Healing of the anterior attachment of the rabbit meniscus to bone. Clinical Orthopaedics, v.348, p.246-258, 1998

HULSE, D.A.; JOHNSON, A.L. Tratamento da doença articular. In: FOSSUM, T.W. Cirurgia de pequenos animais. São Paulo: Rocca, 2002. Cap.30, p.1076-1077.

MCCARTY, E.C. et al. Meniscus repair: considerations in treatment and update of clinical results. Clinical Orthopaedics and Related Research, v.402, n.1, p.122-134, 2002.
PIGOSSI, N. Glicerina na conservação de dura-mater. Estudo experimental. 1967. 86f. Dissertação (Livre Docência) Universidade de São Paulo, São Paulo, SP.

RECKERS, L.J. et al. Effects of different preservation temperatures and periods menisci cellularity in rabbits. Acta Cirúrgica Brasileira, v.20, n.6, p.428-432, 2005. Disponível em: $<$ http://www.scielo.br/scielo.php?script=sci_arttext\&pid=S010286502005000600006\&lng=en\&nrm=iso >. Acesso em: 21 mar. 2011. doi: 10.1590/S0102-86502005000600006.

RIJK, P.C. Meniscal allograft transplantation - Part I: background, results, graft selection and preservation, and surgical considerations. Arthroscopy, v.20, n.7, p.728-743, 2004. Disponível em: <http:// www.sciencedirect.com/science/article/B6WB8-4D8F5K5-S/2/ a660ee9d553c1b5d6a5faa8950acb01a>. Acesso em: 18 mar. 2012. doi: 10.1016/j.arthro.2004.06.015.

SOLOMON, D.H. et al. Does this patient have a torn meniscus or ligament of the knee? Value of the physical examination. Journal of the American Medical Association, v.286, n.13, p.1610-1620, 2001.

TORRES, B.B.J. et al. Como proceder nas lesões meniscais em cães - Revisão. Clínica Veterinária, n.63, p.48-58, 2006.

VILELA, L.M. et al. Estrutura e celularidade de meniscos frescos de coelhos (Oryctolagus cuniculus) e preservados em glicerina. Pesquisa Veterinária Brasileira, v.30, n.4, p.295300, 2010a. Disponível em: <http://www.scielo.br/scielo. php?pid=S0100-736X2010000400002\&script=sci_arttext $>$. Acesso em: 17 jul. 2011.

VILELA, L.M. et al. Propriedades mecânicas de meniscos frescos de coelhos e preservados em glicerina 98\%. Ciência Rural, v.40, n.5, p.1114-1120, 2010b. Disponível em: <http://www.scielo.br/ pdf/cr/v40n5/a583cr2571.pdf>. Acesso em: 7 jun. 2010.

WIRTH, C.J. et al. Long-term results of meniscal allograft transplantation. American Journal of Sports Medicine, v.30, p.174-181, 2002.

WYLAND, D.J. et al. Chondropathy after meniscal tear or partial meniscectomy in a canine model. Journal of Orthopaedic Research, v.20, n.5, p.996-1002, 2002. 